\title{
Source of polarized ions and polarized beams at the NUCLOTRON
}

\author{
V.V. Fimushkin ${ }^{1}$ \\ Joint Institute for Nuclear Research \\ Joliot-Curie 6, 141980 Dubna, Moscow region, Russia \\ E-mail: fimushkin@jinr.ru
}

A.D. Kovalenko, R.A. Kuzyakin, M.V. Kulikov, L.V. Kutuzova, Yu.A. Plis, Yu.V. Prokofichev, V.B. Shutov

Joint Institute for Nuclear Research

Joliot-Curie 6, 141980 Dubna, Moscow region, Russia

\section{A.S. Belov, A.V. Turbabin, and V.N. Zubets}

Institute for Nuclear Research of RAS

Prospect 60letiya Oktyabrya, 7A, 117312, Moscow, Russia

\begin{abstract}
The paper describes the JINR polarized ion source operating by means of the atomic beam method. The results of testing of the plasma ionizer with a storage cell and tuning of high frequency transition units are presented. The source was installed in the linac injector hall of the NUCLOTRON in May 2016. The source was commissioned and used in the NUCLOTRON runs in 2016 and February - March 2017. Polarized and unpolarized deuteron beams as well as polarized proton beam were produced to accelerate in the NUCLOTRON. The polarized deuteron beam with pulsed current up to $3.7 \mathrm{~mA}$ has been produced. Deuteron beam polarization of 0.6-0.9 of theoretical values for different modes of high frequency transition units has been measured with the NUCLOTRON ring internal polarimeter for the accelerated deuteron and proton beams.
\end{abstract}

XVII International Workshop on Polarized Sources, Targets \& Polarimetry

16-20 October 2017

Kaist, South Korea

\section{${ }^{1}$ Speaker}




\section{Introduction}

The high intensity pulsed source of polarized ions (SPI) has been developed at JINR in collaboration with INR RAS for injection of polarized deuterons and protons into the Nuclotron and future collider of heavy and light ions NICA [1]. The SPI is an atomic beam-type polarized ion source with a charge-exchange plasma ionizer and a storage cell in the ionization region [24]. Some parts of the polarized ion source CIPIOS from IUCF [5] are used in the project.

\section{General description of the SPI}

A schematic diagram of the SPI is shown in Figure 1. The source consists of an atomic beam apparatus, the plasma charge-exchange ionizer and the system to transport the polarized ion beam and turn the spin to the vertical direction.

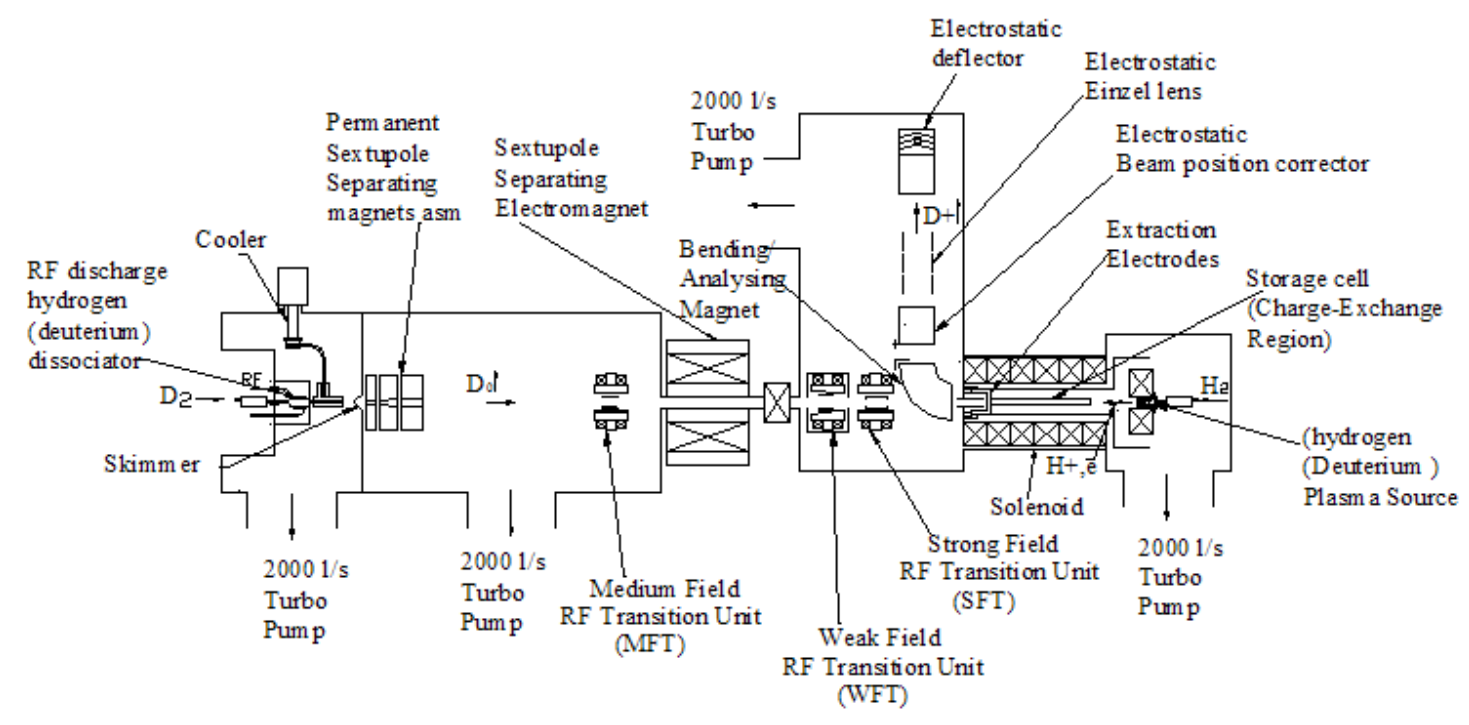

Figure 1. Schematic diagram of the SPI.

\subsection{Atomic beam apparatus}

The polarized deuterium or hydrogen atoms with thermal energy are produced by the atomic beam apparatus consisting of a pulsed radio frequency (RF) discharge dissociator, sextupole magnets and HFT units.

Dissociation of molecular deuterium in RF discharge is used to produce atomic deuterium as in a conventional atomic beam type source. Molecular deuterium is injected by a pulsed electromagnetic gas valve into the dissociator tube where pulsed RF discharge is induced. Deuterium molecules dissociate into atoms in the RF discharge plasma in collisions with plasma electrons. Atomic deuterium flows out into vacuum out from the dissociator tube through Pyrex channel $100 \mathrm{~mm}$ long and 5mm in internal diameter Pyrex channel which ends with a sonic nozzle of $2 \mathrm{~mm}$ in diameter. The walls of the channel are cooled to $\sim 80 \mathrm{~K}$ with the cryocooler (model 350 Cryodyne refrigerator system).

In the initial configuration the sextupole magnet system consisted of the assembly of three permanent sextupoles and one electromagnet sextupole. The sextupole magnet parameters are presented in [5]. The atomic beam passes through the sextupole magnets where atoms in the 
spin states with $m_{j}=1 / 2$ are focused and the ones in the spin state with $m_{j}=-1 / 2$ are defocused. Thus, after passing the sextupole magnets the atoms become polarized.

Nuclear polarization of deuterium atoms being ionized in the strong magnetic field is increased with the system of the HFT units which includes a medium field transition unit (MFT) (installed between the assembly of permanent sextupole magnets and electromagnet sextupole), a weak field (WFT) and a strong field (SFT) transition units which are installed downstream the electromagnet sextupole. For hydrogen atoms alternate turning on of the WFT $(1 \rightarrow 3)$ and the SFT $(2 \rightarrow 4)$ will lead theoretical values of proton vector polarization of -1 or +1 , respectively. We have used MFT, WFT and SFT for polarized deuterium that will provide deuteron vector polarization between +1 and -1 and tensor polarization between +1 and $-2[5,6]$. Many other deuteron polarization states can be produced by using different combinations of the HFT. The sextupole magnets and the HFT units were parts of the CIPIOS designed at IUCF [5].

\subsection{Charge-exchange plasma ionizer with the storage cell}

The polarized atomic deuterium beam produced by the atomic beam apparatus is injected into the storage cell installed inside a solenoid of the charge-exchange plasma ionizer. The atomic beam has duration of up to $3 \mathrm{~ms}$ determined by the pulse duration of the RF discharge dissociator. During the pulse time the polarized deuterium atoms are injected and stored in the cell. Then the hydrogen plasma jet generated by a plasma arc-discharge source is injected into the storage cell in the direction opposite to the deuterium atomic beam through the orifice of $3 \mathrm{~mm}$ in diameter at the end of the storage cell. Polarized deuterons are produced in the storage cell via charge-exchange collisions between polarized deuterium atoms and unpolarized protons:

$\mathrm{D}^{0} \uparrow+\mathrm{H}^{+} \rightarrow \mathrm{D}^{+} \uparrow+\mathrm{H}^{0}$

The following reaction is used to produce polarized protons:

$\mathrm{H}^{0} \uparrow+\mathrm{D}^{+} \rightarrow \mathrm{H}^{+} \uparrow+\mathrm{D}^{0}$

In this case the atomic beam apparatus produces the polarized atomic hydrogen beam and the plasma source produces deuterium plasma.

The cross-section of the charge-exchange reactions (1) and (2) increases while decreasing the relative energy of colliding particles and reaches $5 \cdot 10^{-15} \mathrm{~cm}^{2}$ at the energy of incident particles of $\sim 10 \mathrm{eV}$ (typical for gas discharge plasma).

Radial confinement of the low energy polarized ions formed in the charge-exchange region is provided by the magnetic field of the ionizer solenoid.

Then the polarized ions move slowly under the influence of weak electric fields in plasma in the direction toward the extraction electrode system where they are accelerated to the energy of up to $25 \mathrm{keV}$ together with unpolarized plasma ions. The three electrode Pierce system is used to extract and form the ion beam.

\subsection{Beam transport and spin rotator system}

The extracted $25 \mathrm{keV}$ ion beams pass through the $90^{\circ}$ bending magnet where the polarized ion beam is separated from unpolarized ions. The unpolarized ion beam current is recorded by using the ion beam collector downstream the bending magnet. The polarized beam comes out from the magnet in the vertical direction, passes through the electrostatic einzel lens and then is 
deflected by the $90^{\circ}$ electrostatic deflector into the horizontal plane to $\mathrm{x}$ direction. The direction of the ion spin remains unchanged while passing the deflector. At the source exit the polarized ion beam passes through the solenoid which is used to rotate the deuteron or proton spin to the optimal orientation: this way it is possible to provide the maximum deuteron and proton polarization in the Nuclotron ring. The spatial spin evolution of polarized proton and deuteron beams at the NUCLOTRON injection complex are presented in Figures 2, 3.

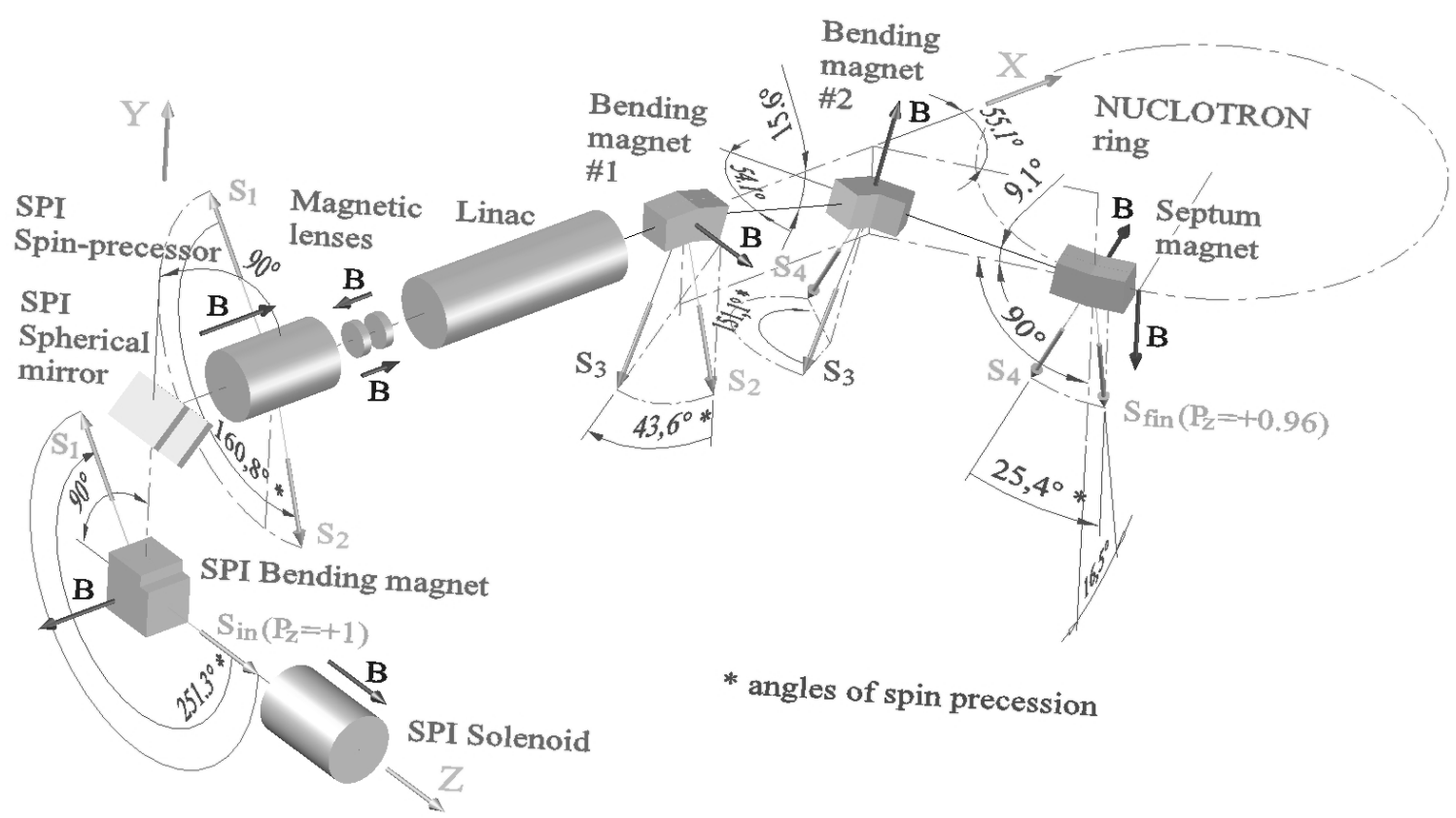

Figure 2. The spatial proton spin evolution at the NUCLOTRON injection complex.

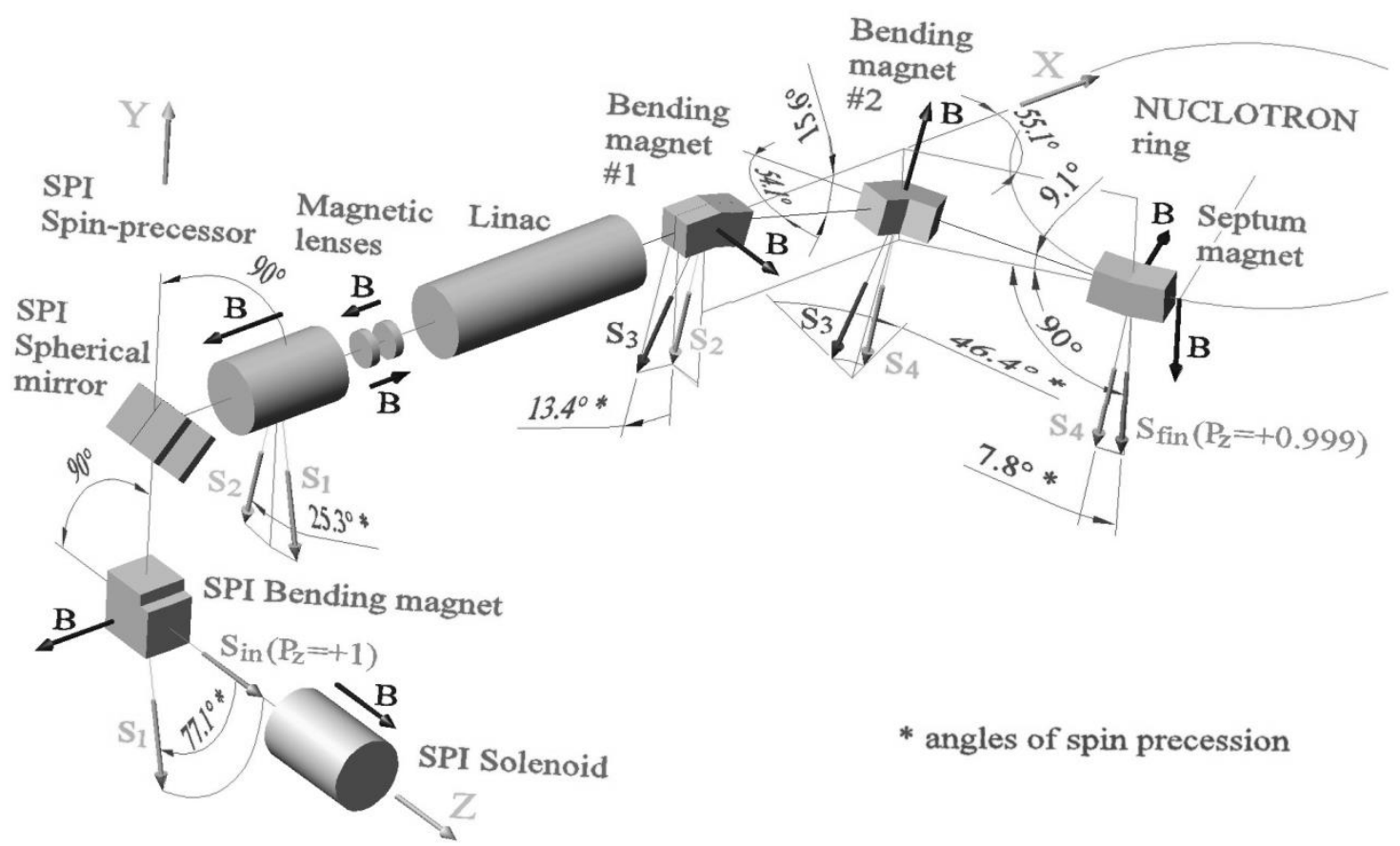

Figure 3. The spatial deuteron spin evolution at the NUCLOTRON injection complex. 


\section{Tests of the SPI with the storage cell and tuning of the HFT units}

\subsection{Tests of the ionizer with the storage cell}

After testing of the SPI with the free polarized atomic deuterium beam in the plasma ionizer described in [7], the storage cell has been installed into the plasma ionizer. The storage cell provides increasing the polarized atoms density in the charge-exchange region by one order of the magnitude in comparison with the free polarized atomic beam. However, even in the pulsed mode of operation the unpolarized ion current which can be transported through the storage cell in the noiseless mode is restricted to $\sim 50 \mathrm{~mA}$ [4]. This method has been tested at INR RAS [3] and the polarized proton beam with the peak current of $11 \mathrm{~mA}$ and polarization of $80 \%$ has been obtained from the source installed at the INR RAS test bench [4].

Initial tests of the SPI with the storage cell in the ionizer region resulted in low intensity of the polarized deuteron beam of $\sim 0.4 \mathrm{~mA}$. It has been found that this of the low intensity polarized deuteron beam was determined in part by low efficiency of collection and extraction of polarized ions from the charge-exchange region inside the storage cell. The collection efficiency has been increased by the growing magnetic field and improvement of its configuration in the charge-exchange region up to $3 \mathrm{kG}$ and increasing the internal diameter of the storage cell. Initially the storage cell had a cylindrical shape of the internal surface with a diameter of $15 \mathrm{~mm}$ and length of $250 \mathrm{~mm}$ (as in ref. [4]). At present the end of the cell directed to the plasma source is closed by diaphragm of $4 \mathrm{~mm}$ in diameter through which the hydrogen plasma is injected into the storage cell. The cell is manufactured from the aluminum alloy.

The latest tests were carried out in the storage cell of a cylindrical form $200 \mathrm{~mm}$ long and with the inner diameter of $18 \mathrm{~mm}$. Besides, the position of the storage cell was shifted by 30 $\mathrm{mm}$ from the extraction system of the polarized deuteron beam. With the storage cell of this geometry the polarized deuteron beam with the peak current of $3 \mathrm{~mA}$ has been obtained from the source (difference of the ion current recorded downstream the analyzing/bending magnet with the polarized atomic deuterium beam "on" and "off"). The unpolarized pulsed proton beam extracted simultaneously with the polarized deuterons was about $70 \mathrm{~mA}$ as well as the beam of $\mathrm{H}_{2}{ }^{+}$ions of $3.8 \mathrm{~mA}$ peak.

The next step to increase the peak current of polarized deuterons was installation of the additional fourth permanent sextupole magnet into the atomic beam apparatus. The parameters of the atomic beam apparatus structure is shown in Table 1.

\begin{tabular}{|l|l|l|l|l|l|}
\hline Magnets & $\begin{array}{l}\text { first } \\
\text { 6-pole }\end{array}$ & $\begin{array}{l}\text { second } \\
\text { 6-pole }\end{array}$ & $\begin{array}{l}\text { third } \\
\text { 6-pole }\end{array}$ & $\begin{array}{l}\text { fourth } \\
\text { 6-pole }\end{array}$ & electromagnet \\
\hline Length, mm & 25 & 47 & 62 & 85 & 230 \\
\hline Input diameter, mm & 9.6 & 14.2 & 18.6 & 29 & 31 \\
\hline Output diameter, mm & 12.8 & 18 & 19.4 & 29 & 31 \\
\hline Magnetic field, T & 1.26 & 1.23 & 1.37 & 1.30 & 0.9 \\
\hline
\end{tabular}

Table 1. Magnetic structure of the SPI atomic beam apparatus.

Usage of the fourth sextupole magnet resulted in increasing the polarized deuteron by $15 \%$. Further optimization of the magnetic field distribution in the ionizer brought the results demonstrated in Figure 4. At the polarized deuteron beam of $3.68 \mathrm{~mA}$ the unpolarized pulsed proton beam is equal to $75 \mathrm{~mA}$ and the beam of $\mathrm{H}_{2}{ }^{+}$ions $-3.8 \mathrm{~mA}$. 


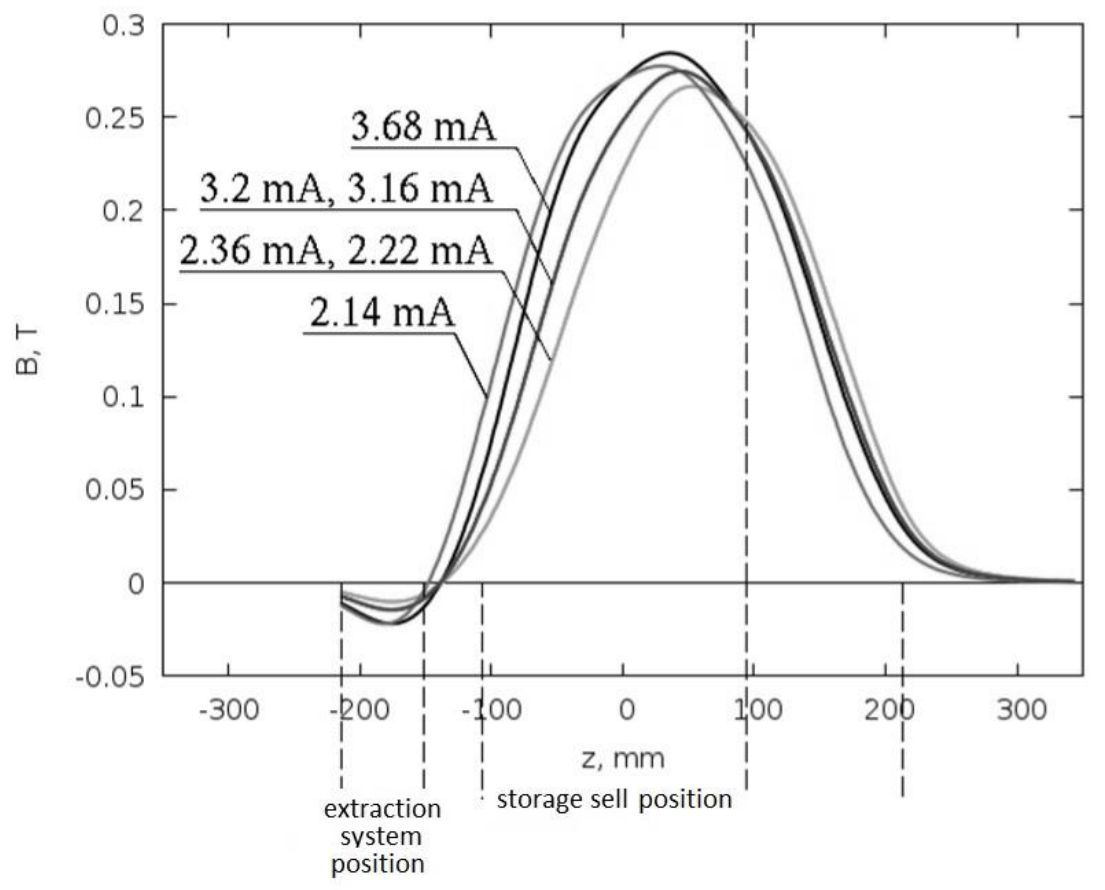

Figure 4. Values of the polarized deuteron peaks in dependence on the ionizer magnetic field.

To estimate the depolarization of the polarized deuteron beam in the ionizer region, we should simulate the following: 1) the time evolution of the spin wave function of the deuterium atomic beam, in particular, near the point where the magnetic field changes sign (zero crossing region) and 2) the same for the polarization of the deuterons produced after ionization. The condition of adiabaticity in the first case is that the angular velocity of the precession $\omega_{p}=$ $2 \mu_{e} B_{r} / 3 \hbar$ should be much more than the angular velocity of the field rotation as it is seen when the particle is moving with velocity $v$ at the distance $r$ from the axis $\omega_{B}=2 v / r$. In our setup this condition is easily fulfilled for thermal atoms as confirmed by the exact solution of the Schrödinger equation. In the second case, we have the "sudden" crossing condition $\omega_{p} / \omega_{B} \ll 1$, where $\omega_{p}=\mu_{d} B_{r} / \hbar$. The solution of the classical equation of the evolution of the deuteron polarization jointly with the Lorentz equations shows that the latter condition is fulfilled for $r \leq 5 \mathrm{~mm}$ while the deuteron energy reducing till $2 \mathrm{eV}$ with polarization loss $\approx 3 \%$.

The beam of $\mathrm{H}_{2}{ }^{+}$ions is not separated from the polarized deuteron beam in the analyzing/bending magnet and in the electrostatic deflector of the SPI. But after accelerating in the linac and passing through a stripping target the unpolarized protons formed due to striping of the $\mathrm{H}_{2}{ }^{+}$ions, are deflected from the polarized deuteron beam entirely in the extraction after the bending magnets. Thus, the unpolarized $\mathrm{H}_{2}{ }^{+}$ions do not dilute the polarized deuteron beam injected into the NUCLOTRON.

\subsection{Measurements of efficiency of the HFT with a Breit - Rabi polarimeter}

HFTs of the SPI were tuned in their operational position by means of the Breit - Rabi polarimeter. A schematic diagram of the ABS components and the polarimeter is shown in Figure 5. 


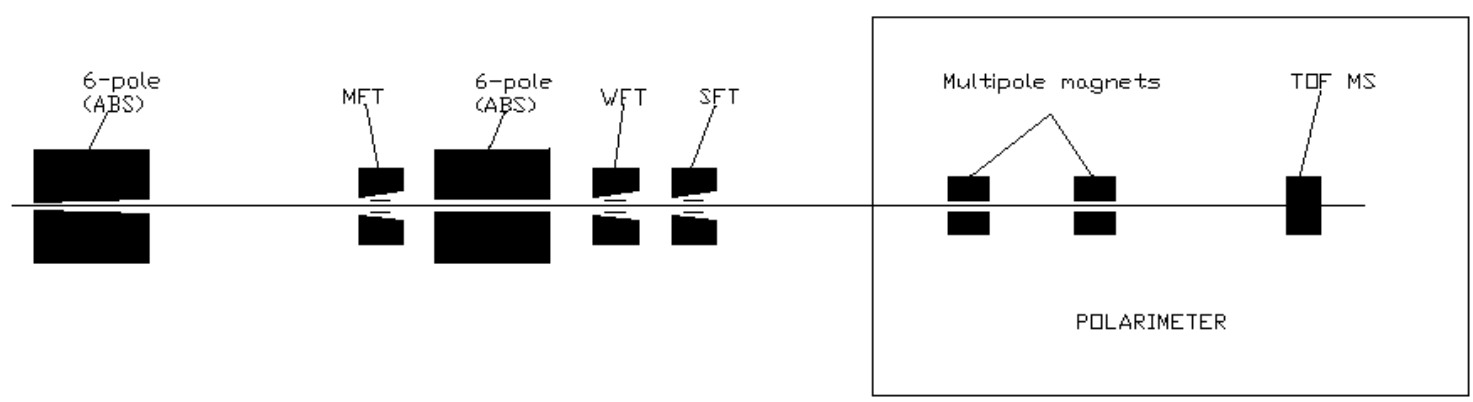

Figure 5. Schematic diagram of the ABS with the Breit - Rabi polarimeter.

Two permanent multipole magnets are used in the polarimeter. Their parameters were determined by race-tracing calculations. The first magnet is a quadrupole with the aperture of $17 \mathrm{~mm}$ and length of $140 \mathrm{~mm}$. The second magnet is a sextupole with the aperture of $18 \mathrm{~mm}$ and length of $125 \mathrm{~mm}$. The maximum magnetic field of the both magnets is $1.6 \mathrm{~T}$. These magnets were installed into the vacuum chamber inside of the ionizer solenoid instead of the storage cell. The time of-flight mass spectrometer (TOF MS) was installed into the vacuum chamber of the plasma source to measure the polarized atomic beam intensity (the plasma source was removed from the chamber during the tuning procedure).

If one of the HFT is "on", then respective part of the atoms under transition between the spin states (with change of their electron spin state in strong magnetic field from $m_{j}=1 / 2$ to $m_{j}=-1 / 2$ ) will be defocused by the multipole magnets of the polarimeter. For deuterium atomic beam this decreases its intensity measured by the TOF MS by one third. Simultaneous activation of two HFT working with $100 \%$ efficiency decreases the deuterium atomic beam intensity by two thirds. The measurements of the relative change of the polarized atomic beam density by the TOF MS with HFT "off" and "on", will allow one to determine efficiencies of the HFT of the ABS. The procedure of tuning the HFT units with the Breit-Rabi polarimeter is given in [8]. The measured efficiencies of other HFT units and their combinations are shown in Table 2.

\begin{tabular}{|c|c|c|c|c|c|c|c|}
\hline & SFT 2-6 & SFT 3-5 & $\begin{array}{c}\text { MFT 3-4 + } \\
\text { SFT 2-6 }\end{array}$ & $\begin{array}{l}\text { MFT 1-4 } \\
+ \text { SFT 2-6 }\end{array}$ & $\begin{array}{l}\text { MFT 1-4 } \\
+ \text { SFT 3-5 }\end{array}$ & WFT 1-4 & $\begin{array}{c}\text { MFT 3-4 } \\
+ \text { WFT 1,2 } \\
-3,4\end{array}$ \\
\hline Efficiency & $0.87 \pm 0.06$ & $0.83 \pm 0.06$ & $0.91 \pm 0.03$ & $0.93 \pm 0.03$ & $0.92 \pm 0.03$ & $0.93 \pm 0.06$ & $0.62 \pm 0.07$ \\
\hline
\end{tabular}

Table 2. The measured efficiencies of the HFT.

The efficiences were calculated taking into account in complete (97\%) defocusing of the atoms under transition with the polarimater multipole magnets into the TOF MS aperture $(27 \mathrm{~mm})$. The defocusing factor by the magnets of the polarimeter was estimated by using MC simulation of the polarized atom tracking through ABS and the polarimeter system. As shown in Table 1 the efficencies of the most HFT are around 90\% except WFT 1,2-3,4. We have planed to rebuild the WFT and improve its efficency.

\section{Commissioning of the SPI}

The SPI has been moved to the linac hall in May 2016. It was commissioned and used in the NUCLOTRON runs of 2016 and February - March 2017. In these runs the SPI operated in 
mode with polarized deuterons during 900 hours and with unpolarized deuterons during 600 hours. Also there was a short test run during 40 hours with polarized protons.

The measurements of the deuteron polarization carried out by means of the internal NUCLOTRON polarimeter [9] resulted in deuteron polarization of $0.6-0.88$ of theoretically maximum values. Tensor deuteron polarization of $0.88 \pm 0.049 ;-1.47 \pm 0.031$ (theoretically maximum $+1,-2$ ) has been measured by additional tuning of the HFTs with the internal polarimeter. The measurements have shown long term stability of polarization. This has demonstrated the lack of depolarization in the plasma ionizer storage cell used for the first time for the polarized ion source operating in continuous accelerator runs. Proton beam polarization of $-0.354 \pm 0.022$ has been measured [10] which is close to the expected polarization of -0.39 with the present beam injection channel between the linac and the NUCLOTRON ring [11].

\section{Conclusions}

The SPI of JINR has been tested with the storage cell installed into the charge-exchange region of the plasma ionizer. The polarized deuteron beam with the pulsed current up to $4 \mathrm{~mA}$ has been produced after studying of the storage cell operation. The HFT units were tuned with by means of the Breit-Rabi polarimeter. The SPI was installed in the linac injector hall of the NUCLOTRON in May 2016. The source was commissioned and used in the NUCLOTRON runs in 2016 and February - March 2017. The polarized and unpolarized deuteron beams were produced as well as polarized protons for acceleration in the NUCLOTRON. The deuteron beam polarization of $0.6-0.88$ of theoretical values for different modes of the HFT units operation has been measured by means the NUCLOTRON ring internal polarimeter for the accelerated deuteron and proton beams.

\section{References}

[1] G.V. Trubnikov, N.N. Agapov, O.I. Brovko, A.V. Butenko, E.D. Donets, A.V. Eliseev, V.V. Fimushkin et al., Proc. of the $4^{\text {th }}$ Int. Particle Accelerator Conf. IPAC2013, TUPFI009 1343 (2013).

[2] A.S. Belov, S.K. Esin, S.A. Kubalov, V.E. Kuzik, A.A. Stepanov, and V.P. Yakushev, Nucl. Instrum. and Meth. In Phys. Res. A255 (1987) 442.

[3] A.S. Belov, S.K. Esin, L.P. Netchaeva, V.S. Klenov, A.V. Turbabin, and G.A. Vasil'ev, Proc. Int. Workshop on Polarized Gas Targets and Polarized Beams, AIP Conf. Proc. 421 (1998) 362.

[4] A.S. Belov, S.K. Esin, L.P. Netchaeva, A.V. Turbabin, and G.A. Vasil'ev, Proc. Int. Symp. on High Energy Spin Physics, (1999) 22.

[5] V.P. Derenchuk and A.S. Belov, Proc. 2001 Particle Accelerator Conf., WPAH008 2093 (2001).

[6] V.V. Fimushkin, A.D. Kovalenko, L.V. Kutuzova, Yu.V. Prokofichev, A.S. Belov, A.V. Turbabin, and V.N. Zubets, Proc. 20th Int. Symp. on High Energy Spin Physics, PEPAN 45 (2014) 297.

[7] V.V. Fimushkin, A.D. Kovalenko, L.V. Kutuzova, Yu.V. Prokofichev, A.S. Belov, A.V. Turbabin, and V.N. Zubets, XVI Workshop on High Energy Spin Physics, IOP Conf. Ser. 678 (2016) 012058.

[8] A. S. Belov, D. E. Donets, V. V. Fimushkin, A. D. Kovalenko, L. V. Kutuzova, Yu. V. Prokofichev, V. B. Shutov, A. V. Turbabin, V. N. Zubets, XVII Workshop on High Energy Spin Physics, IOP Conf. Ser. 938 (2017) 012017. 
[9] Y. Skhomenko, V.P. Ladygin et al., XVII Workshop on High Energy Spin Physics, IOP Conf. Ser. 938 (2017) 012022.

[10] V.P. Ladygin, Yu.V. Gurchin et al., XVII Workshop on High Energy Spin Physics, IOP Conf. Ser. 938 (2017) 012008.

[11] Yu.N. Filatov, A.V. Butenko, A.D. Kovalenko, V.A. Mikhaylov, A.M. Kondratenko, M.A. Kondratenko, XVII Workshop on High Energy Spin Physics, IOP Conf. Ser. 938 (2017) 012018. 